\title{
ERROR BOUNDS FOR A UNIFORM ASYMPTOTIC EXPANSION OF THE LEGENDRE FUNCTION $P_{n}^{-m}(\cosh z)^{*}$
}

\author{
BY \\ P. N. SHIVAKUMAR AND R. WONG ${ }^{1}$ \\ University of Manitoba, Winnipeg, Manitoba, Canada
}

\begin{abstract}
For fixed $m$ with $m+\frac{1}{2}>0$, an asymptotic expansion for large $n$ is derived for the Legendre function $P_{n}^{-m}(\cosh z)$, which is uniformly valid for $z$ in the unbounded interval $[0, \infty)$. Our method is based on an integral representation of this function. The coefficients in the expansion satisfy a recurrence relation. Simple computable bounds are also constructed for the error terms associated with the expansion.
\end{abstract}

1. Introduction. Asymptotic expansions of the Legendre functions $P_{n}^{-m}(\cosh z)$ and $Q_{n}^{-m}(\cosh z)$, for large $n$ and fixed $m$, which are uniformly valid for $z$ in a complex domain containing the interval $0 \leq z<\infty$, can be found in the book of Olver [1, Chap. 12, $\S \S 12$ and 13], where numerical bounds are also provided for the error terms associated with these expansions. Olver's derivation of the expansions, and the construction of error bounds, are based on the asymptotic theory which he has developed for second-order linear differential equations. In a recent paper [4], Ursell has given an alternative derivation of the expansions, using integral representations of the Legendre functions, and has described a new method for constructing error bounds. However, his bounds are not computable, and are valid only for bounded complex $\mathrm{z}$.

In this paper, we are concerned only with the function $P_{n}^{-m}(\cosh z)$, where $z$ is real. Our objective is to construct error bounds, comparable to those given by Olver [1, p. 466, eq. (12.17)], which are both computable and uniformly valid for $z$ in the infinite interval $[0, \infty)$. Like Ursell, our derivation is based on an integral representation of $P_{n}^{-m}(\cosh z)$. Our approach is motivated by the one given by Szegö [3] for the case $m=0$. Our expansion differs from, but is equivalent to, those given by Olver $[1, \mathrm{p}$. 466] and Ursell [4]. More precisely, we show that for $m+\frac{1}{2}>0$ and for any positive

*Received April 14, 1987.

${ }^{1}$ Department of Applied Mathematics, University of Manitoba, Winnipeg, Manitoba, Canada, R3T 2N2. This research was supported in part by the Natural Sciences and Engineering Research Council of Canada under Grants A7899 and A7359.

(C)1988 Brown University 
integer $p$,

$$
P_{n}^{-m}(\cosh z)=\left(\frac{z}{\sinh z}\right)^{1 / 2}\left[\sum_{\nu=0}^{p-1} c_{\nu}(z) \frac{I_{m+\nu}(u z)}{u^{m+\nu}}+\varepsilon_{p}(z, u)\right],
$$

where $u=n+\frac{1}{2}$ and $I_{\nu}(z)$ is the modified Bessel function. The coefficients $c_{\nu}(z)$ are analytic functions of $z$, and can be obtained recursively. The first three are given by

$$
\begin{aligned}
c_{0}(z)=1, \quad c_{1}(z)=\frac{\Gamma\left(m+\frac{3}{2}\right)}{\Gamma\left(m+\frac{1}{2}\right)}\left(m-\frac{1}{2}\right) \frac{1-z \operatorname{coth} z}{2 z}, \\
c_{2}(z)=\frac{\Gamma\left(m+\frac{5}{2}\right)}{\Gamma\left(m+\frac{1}{2}\right)}\left\{\frac{1}{6}\left(m-\frac{1}{2}\right)\left[1+\frac{3}{z^{2}}-\frac{3}{z} \operatorname{coth} z\right]\right. \\
\left.+\frac{1}{8}\left(m-\frac{1}{2}\right)\left(m-\frac{3}{2}\right) \frac{(1-z \operatorname{coth} z)^{2}}{z^{2}}\right\} .
\end{aligned}
$$

The error term $\varepsilon_{p}(z, u)$ satisfies

$$
\left|\varepsilon_{p}(z, u)\right| \leq \frac{\Gamma\left(m+p+\frac{1}{2}\right)}{\Gamma\left(m+\frac{1}{2}\right)} \frac{(2 z)^{p}}{(1+z)^{p}} M_{p} \frac{I_{m+p}(u z)}{u^{m+p}},
$$

for $0 \leq z<\infty$ and $n>-\frac{1}{2}$, where $M_{p}$ is a constant, independent of $u$ and $z$; cf. [1, p. 466, eq. (12.17)]. The numerical values of the first three $M_{p}$ 's are given by

$$
\begin{aligned}
& M_{1}=\left|m-\frac{1}{2}\right|, \\
& M_{2}=\frac{7}{6}\left|m-\frac{1}{2}\right|+\left|m-\frac{1}{2}\right| \cdot\left|m-\frac{3}{2}\right|, \\
& M_{3}=\frac{5}{8}\left|m-\frac{1}{2}\right|+\frac{19}{8}\left|\left(m-\frac{3}{2}\right)\left(m-\frac{1}{2}\right)\right|+\frac{1}{2}\left|\left(m-\frac{5}{2}\right)\left(m-\frac{3}{2}\right)\left(m-\frac{1}{2}\right)\right| .
\end{aligned}
$$

Note that when $m=\frac{1}{2}, \varepsilon_{i}(z, u)=0$ for $i=1,2,3, \ldots$.

Expansion (1.1) can be rearranged to agree with those obtained by Olver and Ursell, but, in doing so, the simple estimate for the remainder given in (1.3) is lost in the process. A more detailed comparison of our result with the corresponding one given by Olver can be found in the last section of the paper.

As regards the other Legendre function $Q_{n}^{-m}(\cosh z)$, our method can be used to derive the corresponding asymptotic expansion in terms of the modified Bessel function $K_{\nu}(z)$, but, unfortunately, we are unable to obtain error bounds similar to those given in (1.3).

2. Derivation of the expansion. For $m+\frac{1}{2}>0$, we have the well-known integral representation

$$
P_{n}^{-m}(\cosh z)=\left(\frac{1}{2 \pi}\right)^{1 / 2} \frac{(\sinh z)^{-m}}{\Gamma\left(m+\frac{1}{2}\right)} \int_{-z}^{z} e^{-u t}(\cosh z-\cosh t)^{m-1 / 2} d t .
$$

Our first objective is to obtain an appropriate series expansion for the above integrand. To do this, we begin with the result [5, p. 140, (3)]

$$
\left(\frac{2}{\pi z}\right)^{1 / 2} \cos \sqrt{z^{2}-2 z \theta}=\sum_{m=0}^{\infty} \frac{\theta^{m}}{m !} J_{m-1 / 2}(z),
$$


which holds for all complex values of $\theta$, where $J_{\nu}(z)$ is the Bessel function of the first kind. Replacing $z$ by $i z$ and $\theta$ by $i \theta,(2.2)$ becomes

$$
\left(\frac{2}{\pi z}\right)^{1 / 2} \cosh \sqrt{z^{2}-2 z \theta}=\sum_{m=0}^{\infty} \frac{(-1)^{m}}{m !} \theta^{m} I_{m-1 / 2}(z) .
$$

Here use has been made of the identity $J_{\nu}(i z)=i^{\nu} I_{\nu}(z)$. Now put $z^{2}-2 z \theta=t^{2}$, and recall the special cases

$$
I_{-1 / 2}(z)=\left(\frac{2}{\pi z}\right)^{1 / 2} \cosh z, \quad I_{1 / 2}(z)=\left(\frac{2}{\pi z}\right)^{1 / 2} \sinh z .
$$

Inserting these in (2.3) gives

$$
2(\cosh z-\cosh t)=\frac{\sinh z}{z}\left(z^{2}-t^{2}\right)\left[1+\sum_{\nu=1}^{\infty}(-1)^{\nu} \phi_{\nu}(z)\left(z^{2}-t^{2}\right)^{\nu}\right],
$$

where

The desired expansion

$$
\phi_{\nu}(z)=\frac{1}{2^{\nu}(\nu+1) !} \frac{I_{\nu+1 / 2}(z)}{z^{\nu} I_{1 / 2}(z)} \text {. }
$$

$$
\begin{aligned}
&(\cosh z-\cosh t)^{m-1 / 2}=\frac{1}{2^{m-1 / 2}}\left(\frac{\sinh z}{z}\right)^{m-1 / 2}\left(z^{2}-t^{2}\right)^{m-1 / 2} \\
& \cdot \sum_{\nu=0}^{\infty}(-1)^{\nu} \psi_{\nu}(z)\left(z^{2}-t^{2}\right)^{\nu}
\end{aligned}
$$

now follows immediately, where the coefficients $\psi_{\nu}(z)$ satisfy the recurrence relation

$$
\psi_{\nu+1}(z)=\sum_{j=0}^{\nu}\left[\left(m-\frac{1}{2}\right)-\frac{j}{\nu+1}\left(m+\frac{1}{2}\right)\right] \phi_{\nu+1-j}(z) \psi_{j}(z),
$$

$\nu=0,1,2, \ldots$, with $\psi_{0}(z)=1$; see [2]. Simple calculation gives

$$
\psi_{1}(z)=-\frac{1}{4}\left(m-\frac{1}{2}\right) \frac{1-z \operatorname{coth} z}{z^{2}}
$$

and

$$
\begin{aligned}
\psi_{2}(z)=\frac{1}{24}\left(m-\frac{1}{2}\right)\left[\frac{1}{z^{2}}\left(1+\frac{3}{z^{2}}\right)\right. & \left.-\frac{3}{z^{3}} \operatorname{coth} z\right] \\
& +\frac{1}{32}\left(m-\frac{1}{2}\right)\left(m-\frac{3}{2}\right)\left(\frac{1-z \operatorname{coth} z}{z^{2}}\right)^{2} .
\end{aligned}
$$

We now introduce the remainder $\Delta_{p}(z, t)$ defined by

$$
\begin{aligned}
{\left[\frac{2(\cosh z-\cosh t)}{z^{2}-t^{2}} \frac{z}{\sinh z}\right]^{m-1 / 2}=\sum_{\nu=0}^{p-1}(-1)^{\nu} \psi_{\nu}(z)\left(z^{2}-t^{2}\right)^{\nu} } & \\
& +(-1)^{p}\left(z^{2}-t^{2}\right)^{p} \Delta_{p}(z, t) .
\end{aligned}
$$

On account of the well-known formula

$$
I_{\nu}(u z)=\frac{2^{-\nu} u^{\nu} z^{-\nu}}{\pi^{1 / 2} \Gamma\left(\nu+\frac{1}{2}\right)} \int_{-z}^{z}\left(z^{2}-t^{2}\right)^{\nu-1 / 2} e^{-u t} d t
$$


we have, upon inserting (2.10) in (2.1),

$$
P_{n}^{-m}(\cosh z)=\left(\frac{z}{\sinh z}\right)^{1 / 2}\left[\sum_{\nu=0}^{p-1} c_{\nu}(z) \frac{I_{m+\nu}(u z)}{u^{m+\nu}}+\varepsilon_{p}(z, u)\right] \text {, }
$$

where

$$
\varepsilon_{p}(z, u)=\frac{(-1)^{p}}{\sqrt{\pi}(2 z)^{m} \Gamma\left(m+\frac{1}{2}\right)} \int_{-z}^{z} e^{-u t}\left(z^{2}-t^{2}\right)^{p+m-1 / 2} \Delta_{p}(z, t) d t
$$

The coefficient $c_{\nu}(z)$ is given explicitly by

$$
c_{\nu}(z)=(-1)^{\nu} \frac{\Gamma\left(m+\nu+\frac{1}{2}\right)}{\Gamma\left(m+\frac{1}{2}\right)}(2 z)^{\nu} \psi_{\nu}(z)
$$

see (1.2).

In the following sections, we shall show that

$$
\left|\Delta_{p}(z, t)\right| \leq M_{p} /(1+z)^{p}, \quad-z \leq t \leq z,
$$

for some numerical quantity $M_{p}$. The first three $M_{p}$ 's are given in (1.4). The desired estimate (1.3) now follows from (2.13) and (2.11).

3. Some preliminary results. To establish the estimate in $(2.15)$, we shall study in great detail the function

$$
\sigma(z, t)=\frac{z}{\sinh z}\left[\frac{\sinh z}{z}-\frac{2(\cosh z-\cosh t)}{z^{2}-t^{2}}\right] .
$$

From (2.4), it is easily seen that $\sigma$ is a function of $z^{2}-t^{2}$ and so can be written as

$$
\sigma=\sigma(s)=\sum_{\nu=1}^{\infty}(-1)^{\nu-1} \phi_{\nu}(z) s^{\nu}, \quad s=z^{2}-t^{2}
$$

By expanding $\cosh z$ and $\cosh t$ into Maclaurin series, we have

$$
\frac{\cosh z-\cosh t}{z^{2}-t^{2}}=\sum_{n=1}^{\infty} \frac{z^{2(n-1)}+z^{2(n-2)} t^{2}+\cdots+t^{2(n-1)}}{(2 n) !} .
$$

Hence, from (3.1), it follows that

$$
1-\sigma=\frac{2 z}{\sinh z} \sum_{n=0}^{\infty} \frac{z^{2 n}+z^{2(n-1)} t^{2}+\cdots+t^{2 n}}{(2 n+2) !} .
$$

The right-hand side can be written as a double sum. Interchanging the order of summations, and re-indexing one of the two sums, we obtain

$$
1-\sigma=\frac{2 z}{\sinh z} \sum_{r=0}^{\infty} \sum_{n=0}^{\infty} \frac{1}{(2 n+2 r+2) !} z^{2 r} t^{2 n}
$$

From (3.3), it also follows that

$$
\frac{\sinh z}{z}-\frac{2(\cosh z-\cosh t)}{z^{2}-t^{2}}=\sum_{n=1}^{\infty} \alpha_{n}
$$


where

The last equation can be written as

$$
\alpha_{n}=\frac{z^{2 n}}{(2 n+1) !}-\frac{z^{2 n}+z^{2(n-1)} t^{2}+\cdots+t^{2 n}}{(2 n+1) !(n+1)}
$$

$$
\begin{aligned}
\alpha_{n}= & \frac{z^{2}-t^{2}}{(2 n+1) !(n+1)}\left\{z^{2(n-1)}+z^{2(n-2)}\left(z^{2}+t^{2}\right)+\cdots\right. \\
& \left.+\left(z^{2(n-1)}+z^{2(n-2)} t^{2}+\cdots+t^{2(n-1)}\right)\right\} \\
= & \frac{1}{(2 n+1) !(n+1)}\left\{n z^{2(n-1)}+(n-1) z^{2(n-2)} t^{2}+\cdots+t^{2(n-1)}\right\} .
\end{aligned}
$$

A combination of (3.1), (3.6) and (3.7) gives

$$
\frac{\sigma}{z^{2}-t^{2}}=\frac{2 z}{\sinh z} \sum_{n=1}^{\infty} \frac{n z^{2(n-1)}+(n-1) z^{2(n-2)} t^{2}+\cdots+t^{2(n-1)}}{(2 n+2) !} .
$$

Analogous to (3.4), the right-hand side of (3.8) can again be viewed as a double sum. Interchanging the order of the summation signs and re-indexing the sums, we obtain

$$
\frac{\sigma}{z^{2}-t^{2}}=\frac{2 z}{\sinh z} \sum_{r=0}^{\infty} \sum_{n=0}^{\infty} \frac{(r+1)}{(2 n+2 r+4) !} z^{2 r} t^{2 n} .
$$

Differentiating (3.5) and (3.9) with respect to $t$, we have, respectively,

$$
\frac{\partial \sigma}{\partial t}=-\frac{2 z}{\sinh z}(2 t) \sum_{r=0}^{\infty} \sum_{n=0}^{\infty} \frac{(n+1)}{(2 n+2 r+4) !} z^{2 r} t^{2 n}
$$

and

$$
\frac{\partial}{\partial t} \frac{\sigma}{z^{2}-t^{2}}=\frac{2 z}{\sinh z}(2 t) \sum_{r=0}^{\infty} \sum_{n=0}^{\infty} \frac{(r+1)(n+1)}{(2 n+2 r+6) !} z^{2 r} t^{2 n} .
$$

LEMMA 1. The function

$$
S(z, t)=\frac{1}{1-\sigma} \frac{\sigma}{z^{2}-t^{2}}
$$

is an even function of $t$, and is decreasing in the interval $0 \leq t \leq z$. Furthermore,

$$
0 \leq S(z, t) \leq \frac{1}{2 z}, \quad 0 \leq t \leq z .
$$

Proof. We first show that on the interval $0 \leq t \leq z, \partial S / \partial t$ is negative. This is equivalent to showing that

$$
(1-\sigma) \frac{\partial}{\partial t} \frac{\sigma}{z^{2}-t^{2}}+\frac{\partial \sigma}{\partial t} \frac{\sigma}{z^{2}-t^{2}}<0 .
$$

Note that if $a_{r n} \geq 0$ and $b_{r n} \geq 0$ for $r$ and $n=0,1,2, \ldots$, then

$$
\left(\sum_{r=0}^{\infty} \sum_{n=0}^{\infty} a_{r n} t^{2 n} z^{2 r}\right)\left(\sum_{r=0}^{\infty} \sum_{n=0}^{\infty} b_{r n} t^{2 n} z^{2 r}\right)=\sum_{r=0}^{\infty} \sum_{n=0}^{\infty} c_{r n} t^{2 n} z^{2 r},
$$

where $c_{r n}$ is the Cauchy product

$$
c_{r n}=\sum_{k=0}^{r} \sum_{j=0}^{n} a_{r-k n-j} b_{k j} .
$$


Hence, from (3.5), (3.9), (3.10) and (3.11), it follows that the quantity on the lefthand side of (3.14) is equal to

where

$$
(2 t)\left(\frac{2 z}{\sinh z}\right)^{2} \sum_{r=0}^{\infty} \sum_{n=0}^{\infty} d_{r n} t^{2 n} z^{2 r},
$$

$$
\begin{aligned}
d_{r n}=\sum_{k=0}^{r} \sum_{j=0}^{n} \frac{1}{(2 n-2 j+2 r-2 k+2) !} \frac{(k+1)(j+1)}{(2 k+2 j+6) !} \\
\quad-\sum_{k=0}^{r} \sum_{j=0}^{n} \frac{(n+1-j)}{(2 n-2 j+2 r-2 k+4) !} \frac{(k+1)}{(2 k+2 j+4) !} .
\end{aligned}
$$

The fact that $d_{r n} \leq 0$, for all nonnegative integers $r$ and $n$, is proved in the appendix. This, of course, establishes the validity of (3.14), and hence

$$
S(z, t) \leq S(z, 0) \text { for } 0 \leq t \leq z .
$$

Now set $t=0$ in (3.4) and (3.8). This gives

$$
S(z, 0)=\sum_{n=1}^{\infty} \frac{n z^{2(n-1)}}{(2 n+2) !} / \sum_{n=0}^{\infty} \frac{z^{2 n}}{(2 n+2) !} .
$$

The sum in the numerator can be written as

$$
\frac{1}{2 z^{3}} \sum_{n=1}^{\infty} \frac{z^{2 n+1}}{(2 n+1) !}-\frac{1}{z^{4}} \sum_{n=1}^{\infty} \frac{z^{2 n+2}}{(2 n+2) !},
$$

which in turn can be expressed as

$$
\frac{1}{2 z^{3}}(\sinh z-z)-\frac{1}{z^{4}}\left(\cosh z-1-\frac{z^{2}}{2}\right) .
$$

The sum in the denominator of (3.17) is clearly $(\cosh z-1) / z^{2}$. Thus

$$
S(z, 0)=\frac{1}{2 z}\left[\frac{z \sinh z-2 \cosh z+2}{z \cosh z-z}\right] .
$$

The quantity inside the square bracket has a positive derivative, and hence is increasing. The limit of this quantity, as $z \rightarrow+\infty$, is 1 . Hence

$$
S(z, 0) \leq \frac{1}{2 z} \text { for } 0<z<\infty .
$$

The second inequality in (3.13) now follows from (3.16) and (3.19). The first inequality in (3.13) is obvious, in view of (3.5) and (3.9). The proof of Lemma 1 is therefore complete.

Replacing $t$ by 0 on the right-hand side of (3.3) gives

$$
\frac{\cosh z-\cosh t}{z^{2}-t^{2}} \geq \frac{1}{z^{2}}(\cosh z-1) \text {. }
$$

Put

$$
\sigma_{1} \equiv 1-\frac{2 z}{\sinh z}\left(\frac{\cosh z-1}{z^{2}}\right) \text {. }
$$

Clearly, from (3.1) and (5.2),

$$
1-\sigma \geq 1-\sigma_{1} \geq 0
$$

The following result is an alternative to Lemma 1 . 
LEMMA $1^{\prime}$. For $-z \leq t \leq z$, we have

$$
0 \leq \frac{1}{1-\sigma_{1}} \cdot \frac{\sigma}{z^{2}-t^{2}} \leq \frac{1}{8}
$$

Proof. From (3.3), it follows that

$$
\frac{\sinh z}{z}-\frac{2(\cosh z-\cosh t)}{z^{2}-t^{2}}=\sum_{n=1}^{\infty} \alpha_{n}
$$

where

which can be written as

$$
\alpha_{n}=\frac{z^{2 n}}{(2 n+1) !}-\frac{z^{2 n}+z^{(2 n-1)} t^{2}+\cdots+t^{2 n}}{(2 n+1) !(n+1)},
$$

$$
\begin{aligned}
\alpha_{n}= & \frac{z^{2}-t^{2}}{(2 n+1) !(n+1)}\left\{z^{2(n-1)}+z^{2(n-2)}\left(z^{2}+t^{2}\right)+\cdots\right. \\
& \left.+\left(z^{2(n-1)}+z^{2(n-2)} t^{2}+\cdots+t^{2(n-1)}\right)\right\} \\
= & \frac{1}{(2 n+1) !(n+1)}\left\{n z^{2(n-1)}+(n-1) z^{2(n-2)} t^{2}+\cdots+t^{2(n-1)}\right\} .
\end{aligned}
$$

Thus

$$
\frac{\sigma}{z^{2}-t^{2}}=\frac{z}{\sinh z} \sum_{n=1}^{\infty} \frac{n z^{2(n-1)}+(n-1) z^{2(n-2)} t^{2}+\cdots+t^{2(n-1)}}{(2 n+1) !(n+1)} .
$$

Replacing $t^{2}$ by $z^{2}$ and summing up $1+2+\cdots+n$ gives

$$
\begin{aligned}
\frac{\sigma}{z^{2}-t^{2}} & \leq \frac{z}{2 \sinh z} \sum_{n=1}^{\infty} \frac{n}{(2 n+1) !} z^{2(n-1)} \\
& =\frac{z}{4 \sinh z} \sum_{n=1}^{\infty}\left[\frac{1}{(2 n) !}-\frac{1}{(2 n+1) !}\right] z^{2(n-1)}
\end{aligned}
$$

which in turn yields

$$
\frac{\sigma}{z^{2}-t^{2}} \leq \frac{z}{4 \sinh z}\left\{\frac{\cosh z}{z^{2}}-\frac{\sinh z}{z^{3}}\right\}
$$

Coupling (3.21) and (3.25), we obtain

$$
\frac{1}{1-\sigma_{1}} \frac{\sigma}{z^{2}-t^{2}} \leq \frac{1}{8} \frac{z \cosh z-\sinh z}{z \cosh z-z} \leq \frac{1}{8}
$$

thus proving Lemma $1^{\prime}$.

LEMMA 2. Let $-z \leq t \leq z$, and put $s=z^{2}-t^{2}$. Then, for any nonnegative integer $n$, we have

$$
\begin{aligned}
\left|\sigma^{(n)}(s)\right| & \leq \frac{1}{(2 z)^{n}}\left[1+\frac{n}{2 !}+\frac{n(n-1)}{3 !}+\cdots+\frac{n !}{(n+1) !}\right] \\
& =\frac{1}{(2 z)^{n}}\left(\frac{2^{n+1}-1}{n+1}\right) .
\end{aligned}
$$


Proof. Differentiating (3.2) term-by-term gives

$$
\sigma^{(n)}(s)=\sum_{\nu=n}^{\infty}(-1)^{\nu} \frac{\nu(\nu-1) \cdots(\nu-n+1)}{(\nu+1) !}(\nu+1) ! \phi_{\nu}(z) s^{\nu-n}
$$

The quotient under the summation sign can be written as

$\frac{1}{(\nu-n+1) !}-\frac{n}{(\nu-n+2) !}+\frac{n(n-1)}{(\nu-n+3) !}+\cdots+(-1)^{n-1} \frac{n(n-1) \cdots 2}{\nu !}+(-1)^{n} \frac{n !}{(\nu+1) !}$. Inserting this in (3.27) yields

$$
\sigma^{(n)}(s)=u+u_{0}+\cdots+u_{n-1}
$$

where

$$
u_{j}=\sum_{\nu=n}^{\infty}(-1)^{\nu+j+1} \frac{n(n-1) \cdots(n-j)}{(\nu-n+2+j) !}(\nu+1) ! \phi_{\nu}(z) s^{\nu-n},
$$

$j=0,1, \ldots, n-1$. A similar expression is given for $u$. From (2.5), it is easily seen that

$$
\begin{aligned}
(\nu+1) ! \phi_{\nu}(z) & =\frac{1}{(2 z)^{\nu} I_{1 / 2}(z)} I_{\nu+1 / 2}(z) \\
& =\frac{\sqrt{z / 2}}{I_{1 / 2}(z)} \frac{1}{z^{2 \nu}} \sum_{p=\nu}^{\infty} \frac{(z / 2)^{2 p}}{\Gamma\left(p+\frac{3}{2}\right)(p-\nu) !} .
\end{aligned}
$$

We now substitute (3.30) in (3.29) and interchange the order of summations. The result is

$u_{j}=(-1)^{j+1} n(n-1) \cdots(n-j) \frac{\sqrt{z / 2}}{I_{1 / 2}(z)} \sum_{p=n}^{\infty} \frac{(z / 2)^{2 p}}{\Gamma\left(p+\frac{3}{2}\right)} \sum_{\nu=n}^{p} \frac{(-1)^{\nu}}{(\nu-n+2+j) !(p-\nu) !} \frac{s^{\nu-n}}{z^{2 \nu}}$.

After re-indexing, the finite sum on the right can be written as

$$
\frac{(-1)^{n-2-j}}{s^{2+j} z^{2(n-2-j)}(p-n+2+j) !} \sum_{l=j+2}^{p-n+2+j}\left(\begin{array}{c}
p-n+2+j \\
l
\end{array}\right)(-1)^{l} \frac{s^{l}}{z^{2 l}} \text {. }
$$

By the binomial theorem, the last sum is equal to

$$
\left(1-\frac{s}{z^{2}}\right)^{p-n+2+j}-1+(p-n+2+j) \frac{s}{z^{2}}-+\cdots+(-1)^{j+2}\left(\begin{array}{c}
p-n+2+j \\
j+1
\end{array}\right) \frac{s^{j+1}}{z^{2(j+1)}} .
$$

Applying Taylor's theorem with derivative form of the remainder to the function $(1-x)^{m}$ with $x=s / z^{2}$ and $m=p-n+2+j$, it is easily seen that the above quantity is bounded by

$$
\left(\begin{array}{c}
p-n+2+j \\
j+2
\end{array}\right) \frac{s^{j+2}}{z^{2(j+2)}}
$$

A combination of these results gives

$$
\left|u_{j}\right| \leq \frac{n(n-1) \cdots(n-j)}{(j+2) !}\left[\frac{\sqrt{z / 2}}{I_{1 / 2}(z)} \sum_{p=n}^{\infty} \frac{(z / 2)^{2 p-2 n}}{2^{2 n} \Gamma\left(p+\frac{3}{2}\right)(p-n) !}\right] .
$$

The infinite series on the right can be summed in closed form, and its value is

$$
\frac{1}{2^{2 n}}\left(\frac{2}{z}\right)^{n+1 / 2} I_{n+1 / 2}(z) \text {. }
$$


Since $I_{n+1 / 2}(z) \leq I_{1 / 2}(z)$, we obtain

$$
\left|u_{j}\right| \leq \frac{n(n-1) \cdots(n-j)}{(j+2) !} \frac{1}{(2 z)^{n}} .
$$

By the same argument, we also have

$$
|u| \leq \frac{1}{(2 z)^{n}} .
$$

The desired result (3.26) now follows from (3.28), (3.32) and (3.33).

Lemma $2^{\prime}$. Let $-t \leq z \leq t$ and put $s=z^{2}-t^{2}$. Then for any nonnegative integer $n$, we have

$$
\left|\sigma^{(n)}(s)\right| \leq \frac{\Gamma\left(\frac{3}{2}\right)}{2^{2 n} \Gamma\left(n+\frac{3}{2}\right)}\left(\frac{2^{n+1}-1}{n+1}\right) .
$$

Proof. The argument here proceeds in exactly the same manner as in the proof of Lemma 2 until we reach equation (3.31). By comparing the coefficients in the last power series with those in

$$
\left(\frac{z}{2}\right)^{-1 / 2} I_{1 / 2}(z)=\sum_{s=0}^{\infty} \frac{(z / 2)^{2 s}}{\Gamma\left(s+\frac{3}{2}\right) s !},
$$

it is evident that the quantity inside the square bracket in (3.31) is less than

$$
\frac{1}{2^{2 n}} \frac{\Gamma\left(\frac{3}{2}\right)}{\Gamma\left(n+\frac{3}{2}\right)} \text {. }
$$

Therefore, for $j=0,1, \ldots, n-1$,

$$
\left|u_{j}\right| \leq \frac{n(n-1) \cdots(n-j)}{(j+2) !} \frac{\Gamma\left(\frac{3}{2}\right)}{2^{2 n} \Gamma\left(n+\frac{3}{2}\right)} .
$$

By the same argument, we also have

$$
|u| \leq \frac{\Gamma\left(\frac{3}{2}\right)}{2^{2 n} \Gamma\left(n+\frac{3}{2}\right)} .
$$

The desired result (3.34) now follows from (3.28), (3.35), (3.36) and the equality in (3.26).

For $z \geq 1$, we have from Lemma 2

$$
\left|\sigma^{(n)}(s)\right| \leq \frac{1}{(1+z)^{n}}\left(\frac{2^{n+1}-1}{n+1}\right),
$$

and, for $0 \leq z \leq 1$, we have from Lemma $2^{\prime}$,

$$
\left|\sigma^{(n)}(s)\right| \leq \frac{1}{(1+z)^{n}} \frac{\Gamma\left(\frac{3}{2}\right)}{2^{n} \Gamma\left(n+\frac{3}{2}\right)}\left(\frac{2^{n+1}-1}{n+1}\right) .
$$

Consequently,

$$
\left|\sigma^{(n)}(s)\right| \leq \frac{1}{(1+z)^{n}}\left(\frac{2^{n+1}-1}{n+1}\right)
$$

for all $z \geq 0$. 
4. Proof of (2.15). By the binomial theorem,

$$
(1-\sigma)^{m-1 / 2}=\sum_{r=0}^{p-1} \frac{\Gamma\left(\frac{1}{2}-m+r\right)}{r ! \Gamma\left(\frac{1}{2}-m\right)} \sigma^{r}+\sigma^{p} R_{p}^{(2)},
$$

where

$$
R_{p}^{(2)}=\frac{\Gamma\left(\frac{1}{2}-m+p\right)}{(p-1) ! \Gamma\left(\frac{1}{2}-m\right)} \int_{0}^{1}(1-u)^{p-1}(1-\sigma u)^{m-1 / 2-p} d u .
$$

Replacing each $\sigma^{r}, r=0,1, \ldots, p-1$, on the right-hand side of (4.1) by its Taylor polynomial, we get

$$
(1-\sigma)^{m-1 / 2}=\sum_{j=0}^{p-1}\left(\sum_{r=0}^{p-1} \frac{\Gamma\left(\frac{1}{2}-m+r\right)}{r ! \Gamma\left(\frac{1}{2}-m\right)} \sigma_{r}^{(j)}(0)\right) \frac{s^{j}}{j !}+\frac{s^{p}}{p !} R_{p}^{(1)}+\sigma^{p} R_{p}^{(2)},
$$

where $\sigma_{r}(s) \equiv \sigma^{r}(s)$ and

$$
R_{p}^{(1)}=\sum_{r=0}^{p-1} \frac{\Gamma\left(\frac{1}{2}-m+r\right)}{r ! \Gamma\left(\frac{1}{2}-m\right)} \sigma_{r}^{(p)}(\xi)
$$

$\xi$ being between 0 and $s$. Note that $(1-\sigma)^{m-1 / 2}$ also has the power series expansion given by (2.6). Comparing (4.3) with that series, we conclude that the finite sum in (4.3) must agree with the first $n$ terms in (2.6). Thus

$$
(1-\sigma)^{m-1 / 2}=\sum_{\nu=0}^{p-1}(-1)^{\nu} \psi_{\nu}(z) s^{\nu}+\frac{s^{p}}{p !} R_{p}^{(1)}+\sigma^{p} R_{p}^{(2)} .
$$

From (2.10), it now follows that

$$
\left|\Delta_{p}(z, t)\right| \leq \frac{1}{p !}\left|R_{p}^{(1)}\right|+\left(\frac{\sigma}{z^{2}-t^{2}}\right)^{p}\left|R_{p}^{(2)}\right| .
$$

Since $\sigma_{r}^{(p)}(s)$ can be expressed as a finite sum of products of $\sigma$ and $\sigma^{(j)}, j=1, \ldots, p$, it follows from (3.39) that there is a computable number $M_{p}^{(1)}$ such that

$$
\left|R_{p}^{(1)}\right| \leq \sum_{r=0}^{p-1} \frac{\Gamma\left(\frac{1}{2}-m+r\right)}{r ! \Gamma\left(\frac{1}{2}-m\right)}\left|\sigma_{r}^{(p)}(\xi)\right| \leq M_{p}^{(1)} /(1+z)^{p} .
$$

The first few $M_{p}^{(1)}$ 's are given by

$$
\begin{aligned}
& M_{1}^{(1)}=0, \quad M_{2}^{(1)}=\frac{7}{3}\left|\frac{1}{2}-m\right|, \\
& M_{3}^{(1)}=\frac{15}{4}\left|m-\frac{1}{2}\right|+\frac{57}{4}\left|m-\frac{3}{2}\right|\left|m-\frac{1}{2}\right| .
\end{aligned}
$$

To obtain a bound for $R_{p}^{(2)}$, we first observe that since $0<\sigma<1$, we have $1-u \leq$ $1-\sigma u$. Hence,

$$
\int_{0}^{1}(1-u)^{p-1}(1-\sigma u)^{m-1 / 2-p} d u \leq \int_{0}^{1}(1-\sigma u)^{m-3 / 2} d u \leq \frac{1}{1-\sigma}
$$


for $m+\frac{1}{2}>0$. In view of (3.22), it follows from (4.2) that

$$
\left|R_{p}^{(2)}\right| \leq M_{p}^{(2)} /\left(1-\sigma_{1}\right)
$$

where

$$
M_{p}^{(2)}=\frac{\Gamma\left(\frac{1}{2}-m+p\right)}{(p-1) ! \Gamma\left(\frac{1}{2}-m\right)} .
$$

An alternative bound for $R_{p}^{(2)}$ can be constructed as follows: since $(1-\sigma)^{p} \leq(1-\sigma)$ for $p \geq 1$, (4.9) gives

$$
\int_{0}^{1}(1-u)^{p-1}(1-\sigma u)^{m-1 / 2-p} d u \leq \frac{1}{(1-\sigma)^{p}}
$$

Consequently, we get from (4.2)

$$
\left|R_{p}^{(2)}\right| \leq M_{p}^{(2)} /(1-\sigma)^{p} .
$$

By Lemma 1 ,

$$
\left(\frac{\sigma}{z^{2}-t^{2}}\right)^{p}\left|R_{p}^{(2)}\right| \leq M_{p}^{(2)} /(2 z)^{p},
$$

and by Lemma $1^{\prime}$

$$
\left(\frac{\sigma}{z^{2}-t^{2}}\right)^{p}\left|R_{p}^{(2)}\right| \leq M_{p}^{(2)} / 8^{p}
$$

For $z \geq 1,(4.14)$ implies

$$
\left(\frac{\sigma}{z^{2}-t^{2}}\right)^{p}\left|R_{p}^{(2)}\right| \leq M_{p}^{(2)} /(1+z)^{p}
$$

and for $0 \leq z \leq 1,(4.15)$ yields

$$
\left(\frac{\sigma}{z^{2}-t^{2}}\right)^{p}\left|R_{p}^{(2)}\right| \leq M_{p}^{(2)} /\left(2^{2 p}(1+z)^{p}\right)
$$

Since the right-hand side of (4.16) is greater than the right-hand side of (4.17), we have

$$
\left(\frac{\sigma}{z^{2}-t^{2}}\right)^{p}\left|R_{p}^{(2)}\right| \leq M_{p}^{(2)} /(1+z)^{p}
$$

for all $z \geq 0$.

A combination of (4.6), (4.7) and (4.18) gives

$$
\left|\Delta_{p}(z, t)\right| \leq M_{p} /(1+z)^{p}
$$

with $M_{p}=\frac{1}{p !} M_{p}^{(1)}+M_{p}^{(2)} ;$ cf. (2.15). The desired result now follows from (2.13) and (2.11).

5. Comparison with Olver's result. To compare our results with earlier ones, we first recall the expansion given by Olver [1, Chap. 12, §12]

$$
\begin{aligned}
P_{n}^{-m}(\cosh z)=\frac{1}{u^{m}}\left(\frac{z}{\sinh z}\right)^{1 / 2} & \left\{I_{m}(u z) \sum_{s=0}^{p} \frac{A_{s}^{-m}\left(z^{2}\right)}{u^{2 s}}\right. \\
+ & \left.\frac{z}{u} I_{m-1}(u z) \sum_{s=0}^{p-1} \frac{B_{s}^{-m}\left(z^{2}\right)}{u^{2 s}}+\eta_{2 p+1,1}\left(u, z^{2}\right)\right\}
\end{aligned}
$$


where

$$
\begin{gathered}
\left|\eta_{2 p+1,1}\left(u, z^{2}\right)\right| \leq \lambda_{1}(m) I_{m}(u z) \exp \left[\frac{\lambda_{1}(m)}{u} V_{0, z}\left\{z B_{0}^{-m}\left(z^{2}\right)\right\}\right] \frac{V_{0, z}\left\{z B_{p}^{-m}\left(z^{2}\right)\right\}}{u^{2 p+1}} \\
\lambda_{1}(m)=\sup _{x \in(0, \infty)}\left\{2 x I_{m}(x) K_{m}(x)\right\}
\end{gathered}
$$

and where $V_{a, b}\{f\}$ denotes the total variation of $f$ over the interval $(a, b)$. The coefficients $A_{s}^{-m}(\zeta)$ and $B_{s}^{-m}(\zeta)$ are determined successively by

$$
\begin{aligned}
& B_{s}^{-m}(\zeta)=-A_{s}^{-m^{\prime}}(\zeta)+\frac{1}{\zeta^{1 / 2}} \int_{0}^{\zeta}\left[\frac{4 m^{2}-1}{16}\left\{\operatorname{csch}^{2}\left(v^{1 / 2}\right)-\frac{1}{v}\right\} A_{s}^{-m}(v)\right. \\
& \left.+\left(m-\frac{1}{2}\right) A_{s}^{-m^{\prime}}(v)\right] \frac{d v}{v^{1 / 2}} \\
& A_{s+1}^{-m}(\zeta)=-m\left\{B_{s}^{-m}(\zeta)+B_{s}^{-m}(0)\right\}-\zeta B^{-m^{\prime}}(\zeta) \\
& +\frac{4 m^{2}-1}{16} \int_{0}^{\zeta}\left\{\operatorname{csch}^{2}\left(v^{1 / 2}\right)-\frac{1}{v}\right\} B_{s}^{-m}(v) d v,
\end{aligned}
$$

and $A_{0}^{-m}(\zeta) \equiv 1$. In particular,

$$
\begin{gathered}
B_{0}^{-m}\left(z^{2}\right)=\frac{1-4 m^{2}}{8 z}\left(\operatorname{coth} z-\frac{1}{z}\right) \\
A_{1}^{-m}\left(z^{2}\right)=\frac{1}{2} z^{2}\left\{B_{0}^{-m}\left(z^{2}\right)\right\}^{2}-\left(m-\frac{1}{2}\right) B_{0}^{-m}\left(z^{2}\right)-\xi\left(z^{2}\right)-\frac{1}{6} m\left(\frac{1}{4}-m^{2}\right)
\end{gathered}
$$

where

$$
\xi\left(z^{2}\right)=\frac{4 m^{2}-1}{16}\left(\frac{1}{\sinh ^{2} z}-\frac{1}{z^{2}}\right)
$$

cf. [1, Chap. 12, Ex. 12.1].

An advantage of Olver's expansion (5.1) is that it involves only two Bessel functions $I_{m}$ and $I_{m-1}$, whereas our expansion (1.1) involves all the Bessel functions $I_{m+\nu}$, $\nu=0,1,2, \ldots$ A disadvantage of Olver's expansion is the difficulty in the calculation of the coefficients $A_{s}^{-m}\left(z^{2}\right)$ and $B_{s}^{-m}\left(z^{2}\right)$ given in (5.4) and (5.5). Although there is an alternative way of evaluating these coefficients as outlined in [1, Chap. 12, Ex. 5.2], it still seems simpler to calculate the coefficients $c_{s}(z)$ given in (2.14). Another point which deserves mentioning is that for small $z$, the coefficient $c_{s}(z)$ is $O\left(z^{s}\right)$. Thus the terms in (1.1) are smaller than the corresponding terms in (5.1). As we have mentioned in $\S 1$, it is possible to rearrange our expansion (1.1) so that it agrees with Olver's expansion in (5.1), but the simplicity of the error estimate given in (1.3) will be lost in the process.

To assess our error bound (1.3), we examine the ratio $R_{p}^{-m}(z, u)$ of this bound to the first neglected term $c_{p}(z) I_{m+p}(u z) / u^{m+p}$. After cancelling a common factor, this ratio simplifies to

$$
R_{p}^{-m}(z, u)=\frac{M_{p}(2 z)^{p} /(1+z)^{p}}{(-1)^{p}(2 z)^{p} \psi_{p}(z)}
$$


where $\psi_{p}(z)$ is defined recursively in (2.7). Explicit formulas for $\psi_{1}$ and $\psi_{2}$ are given in (2.8) and (2.9). For any specific value of $m$, the maximum value of $(2 z)^{p}\left|\psi_{p}(z)\right|$ in $0 \leq z<\infty$ can be computed numerically. Since $\psi_{p}(z)$ is analytic in $0 \leq z<\infty$ and $\psi_{p}(z)=O\left(z^{-p}\right)$ as $z \rightarrow \infty$, this value is a finite number. On the other hand, the maximum value of $(2 z)^{p} /(1+z)^{p}$ in $0 \leq z<\infty$ is $2^{p}$. Thus, if $\tilde{R}_{p}^{-m}$ denotes the ratio of the maxima of the absolute values of the numerator and the denominator in (5.9) over the interval $0 \leq z<\infty$, then $\tilde{R}_{p}^{-m}$ is finite for each $p \geq 1$. Furthermore, it can easily be verified that

$$
\tilde{R}_{p}^{-m}=O(1) \quad \text { as } m \rightarrow \infty .
$$

From (5.9), it is also evident that the error bound in (1.3) mimics the behavior of the first neglected term near both $z=0$ and $z=\infty$. Therefore, our bound shares similar features with that of Olver, whereas Ursell's bound [4] breaks down when $z$ becomes unbounded.

Direct comparison between our error bound (1.3) and Olver's bound (5.2) is difficult, since the forms of these bounds are different, and so are the two expansions (1.1) and (5.1). The closest comparison that we can give is to take the one-term approximation given in each of the expansions (1.1) and (5.1). With $p=1$ in (1.1), our expansion gives

$$
P_{n}^{-m}(\cosh z)=\left(\frac{z}{\sinh z}\right)^{1 / 2}\left[\frac{I_{m}(u z)}{u^{m}}+\varepsilon_{1}(z, u)\right],
$$

where, since $z \leq 1+z$,

$$
\left|\varepsilon_{1}(z, u)\right| \leq 2\left|m^{2}-\frac{1}{4}\right| \frac{I_{m+1}(u z)}{u^{m+1}} .
$$

From [1, Chap. 12, Ex. 12.1], we have $V_{0, \infty}\left\{z B_{0}^{-m}\left(z^{2}\right)\right\}=\frac{1}{2}\left|m^{2}-\frac{1}{4}\right|$. Thus, Olver's expansion gives

$$
P_{n}^{-m}(\cosh z)=\left(\frac{z}{\sinh z}\right)^{1 / 2}\left[\frac{I_{m}(u z)}{u}+\frac{1}{u^{m}} \eta_{1,1}\left(u, z^{2}\right)\right]
$$

with

$$
\frac{1}{u^{m}}\left|\eta_{1,1}\left(u, z^{2}\right)\right| \leq \frac{1}{2} \lambda_{1}(m)\left|m^{2}-\frac{1}{4}\right| \exp \left\{\frac{\lambda_{1}(m)}{2 u}\left|m^{2}-\frac{1}{4}\right|\right\} \frac{I_{m}(u z)}{u^{m+1}} .
$$

Since $\lambda_{1}(m) \geq 1$ and $I_{m}(u z) \geq I_{m+1}(u z)$, due to the exponential factor, the right-hand side of $(5.14)$ is greater than the corresponding side of (5.12) for large or moderately large values of $m$.

Acknowledgment. We would like to thank Professor F. W. J. Olver for several helpful discussions on this problem, and the referees for their useful suggestions.

Appendix. In this appendix, we shall prove that the number

$$
\begin{aligned}
d_{r n}=\sum_{k=0}^{r} \sum_{j=0}^{n} \frac{(k+1)(j+1)}{(2 n-2 j+2 r-2 k+2) !} \frac{1}{(2 j+2 k+6) !} \\
\quad-\sum_{k=0}^{r} \sum_{j=0}^{n} \frac{(n+1-j)}{(2 n-2 j+2 r-2 k+4) !} \frac{(k+1)}{(2 j+2 k+4) !}
\end{aligned}
$$


in (3.15) is negative. Since the first double sum on the right-hand side involves $j+1$, replacing the lower limit $j=0$ by $j=-1$ does not change the value of $d_{r n}$. By a similar argument, we may replace the upper limit $j=n$ in the second sum by $j=n+1$. Now we re-index the first double sum by writing $j$ for $j+1$. This gives

$$
d_{r n}=\sum_{k=0}^{r} \sum_{j=0}^{n+1} \frac{(2 j-n-1)(k+1)}{(2 n-2 j+2 r-2 k+4) !(2 j+2 k+4) !} .
$$

With $j+k=l$, (A.2) can also be expressed as

$$
d_{r n}=\sum_{k=0}^{r} \sum_{l=k}^{n+k+1} \frac{a_{k l}}{f(l)}
$$

where

$$
a_{k l}=(2 l-2 k-n-1)(k+1), \quad f(l)=(2 n+2 r-2 l+4) !(2 l+4) ! .
$$

Note that

$$
f(n+r-l)=f(l) .
$$

From (A.1), it is easily seen that $d_{r n}=d_{n r}$ for all $r, n=0,1,2, \ldots$ Thus, without loss of generality, we may assume that $n \geq r$. The remainder of the proof consists of two cases: (i) $n+r$ even, and (ii) $n+r$ odd. Since the arguments in both cases are parallel, we shall present only the details for case (i). First, we split the inner sum in (A.3) into two with one extending over $l=k$ to $l=\frac{1}{2}(n+r)$ and the other over $l=\frac{1}{2}(n+r)+1$ to $l=n+k+1$. Next, we let $l=n+r-l^{\prime}$ and $k=r-k^{\prime}-1$ in the second resulting sum; after this, we write $l$ for $l^{\prime}$ and $k$ for $k^{\prime}+1$. In view of (A.5), this yields

$$
\begin{aligned}
d_{r n}=\sum_{k=0}^{r} \sum_{l=k}^{\frac{1}{2}(n+r)-1} \frac{1}{f(l)}\left[a_{k l}\right. & \left.+a_{r-k n+r-l}\right] \\
& +\sum_{k=0}^{r} \frac{1}{f(k-1)} a_{r-k n+r-k+1}+\sum_{k=0}^{r} \frac{1}{f\left[\frac{1}{2}(n+r)\right]} a_{k \frac{1}{2}(n+r)} .
\end{aligned}
$$

The double sum on the right is expressible as

$$
\sum_{k=0}^{r} \sum_{l=k}^{r}+\sum_{k=0}^{r} \sum_{l=r+1}^{\frac{1}{2}(n+r)-1}
$$

Interchanging the order of summation signs in both terms gives

$$
d_{r n}=\sum_{l=0}^{r} A_{l}+\sum_{l=r+1}^{\frac{1}{2}(n+r)-1} \frac{1}{f(l)} B_{l}+\frac{1}{f\left[\frac{1}{2}(n+r)\right]} C,
$$


where

$$
\begin{aligned}
A_{l} & =\frac{1}{f(l)} \sum_{k=0}^{l}\left(a_{k l}+a_{r-k} n+r-l\right)+\frac{1}{f(l-1)} a_{r-l n+r-l+1} \\
B_{l} & =\sum_{k=0}^{r}\left(a_{k l}+a_{r-k} n+r-l\right) \\
C & =\sum_{k=0}^{r} a_{k \frac{1}{2}(n+r)} .
\end{aligned}
$$

To prove that the numbers $d_{r n}$ are negative, it suffices to show that

$$
\sum_{l=0}^{r} A_{l}<0, \quad B_{l}<0, \text { and } C<0 .
$$

From (A.4), it is easily shown that

$$
a_{k l}+a_{r-k n+r-l}=-4(k+1)^{2}+(4 l-2 n+2 r+8)(k+1)+(r+2)(n-2 l-3) .
$$

Hence

$$
\begin{aligned}
\sum_{k=0}^{l}\left(a_{k l}+a_{r-k} n+r-l\right. & =-\frac{1}{3}(l+1)(l+2)(3 r+3-2 l) \\
\sum_{k=0}^{r}\left(a_{k l}+a_{r-k n+r-l}\right) & =-\frac{1}{3}(r+1)(r+2)(r+3), \\
\sum_{k=0}^{r} a_{k \frac{1}{2}(n+r)} & =-\frac{1}{6}(r+1)(r+2)(r+3) .
\end{aligned}
$$

The last two inequalities in (A.10) now follow immediately from (A.12) and (A.13).

To prove the first inequality in (A.10), we note that

$$
a_{r-l n+r-l+1}=(r-l+1)(n+1),
$$

which, together with (A.7) and (A.11), gives

$$
\begin{aligned}
\sum_{l=0}^{r} A_{l}=-\frac{1}{3} \sum_{l=0}^{r} \frac{(l+1)(l+2)(3 r+3-2 l)}{f(l)}+\sum_{l=0}^{r} \frac{(n+1)(l+1)(r-l)}{f(l)} \\
+\sum_{l=0}^{r} \frac{(n+1)(r-l+1)}{f(l-1)} .
\end{aligned}
$$

In the second sum on the right, we may replace the limits of summation $l=0$ and $l=r$ by $l=-1$ and $l=r-1$, respectively, without changing the value of the sum. After this change, we re-index the summation by writing $l$ for $l+1$. The last two sums are then added together. This yields

$$
\sum_{l=0}^{r} A_{l}=\sum_{l=0}^{r}\left[\frac{(n+1)(r-l+1)(l+1)}{f(l-1)}-\frac{(l+1)(l+2)(3 r+3-2 l)}{3 f(l)}\right] .
$$


Now it can be shown directly that each term under the summation on the right-hand side is negative. This establishes the first inequality in (A.10), and completes the proof of our claim.

\section{REFERENCES}

[1] F. W. J. Olver, Asymptotics and Special Functions, Academic Press, New York, 1974

[2] M. Pourahmadi, Taylor expansion of $\exp \left(\sum_{k=0}^{\infty} a_{k} z^{k}\right)$ and some applications, Amer. Math. Monthly 91, 303-308 (1984)

[3] G. Szegö, Uber einige asymptotische Entwicklungen der Legendreschen Funktionen, Proc. Lond. Math. Soc., (2), 36, 427-450 (1932)

[4] F. Ursell, Integrals with a large parameter: Legendre functions of large degree and fixed order, Math. Proc. Camb. Phil. Soc. 95, 367-380 (1984)

[5] G. N. Watson, A Treatise on the Theory of Bessel Functions, Cambridge University Press, Cambridge, 1944 